\title{
Impaired Bronchoprotection Is Not Induced by Increased Smooth Muscle Mass in Chronic Treatment In Vivo with Formoterol in Asthmatic Mouse Model
}

\author{
W Luo ${ }^{1}$, C-T Liu 1 , Q-H Yang 2 , Q Yu ${ }^{3}$, T Wang ${ }^{4}$
}

\begin{abstract}
Objective: Inhaling $\beta_{2}$-adrenoceptor agonist is first-line asthma treatment, which is used for both acute relief and prevention of bronchoconstriction. However, chronic use of $\beta$-agonists results in impaired bronchoprotection and increasing occurrences of severe asthma exacerbation, even death in clinical practice. The mechanism of $\beta$-adrenoceptor hyposensitivity has not been thoroughly elucidated thus far. Bronchial smooth muscle contraction induces airway narrowing and also mediates airway inflammation. Moreover, bronchial smooth muscle mass significantly increases in asthmatics. We aimed to establish an asthmatic model that demonstrated that formoterol induced impaired bronchoprotection and to see whether increased smooth muscle mass played a role in it.

Methods: We combined routine allergen challenging (seven week sivith repeated application of formoterol, formoterol plus budesonide or physiological saline in allergen-sensitized BALB/c mouse. The bronchoprotection mediated by $\beta$-agonist was measured in five consecutive weels. Smooth muscle mass was shown by morphometric analysis, and $\alpha$-actin expression was detected by western blot.

Results: The trend of bronchoprotection was wavy in drug interventional groups, which initially increased and then decreased. Chronic treatment with formoterol significantly impaired bronchoprotection. According to the morphometric analysis and $\alpha$-actin expression, no significant difference was detected in smooth muscle mass in all groups.

Conclusion: This experiment successfully established that a chronic asthmatic mouse model, which manifested typical features of asthmatic patients, with chronic use of formoterol, result in a loss of bronchoprotection. No significant difference was detected in smooth muscle mass in all groups, which implied some subcellular signalling changes may be the key points.
\end{abstract}

Keywords: $\alpha$-actin, asthma, bronchoprotection, formoterol

WIMJ Open 2014; 1 (3): 78

\section{INTRODUCTION}

Agonists of $\beta$-adrenoceptor might be the most extensively prescribed therapeutic drugs for the treatment of asthma in clinical practice. They are powerful bronchodilators that provide relief of smooth muscle bronchospasm and hence attenuate airway obstruction. In addition to exerting bronchodilation, $\beta$-agonists also mediate bronchoprotection which is defined as inhibition of induced bronchoconstriction

From: ${ }^{1}$ Department of Respiratory Medicine, West China Hospital, Sichuan University, Chengdu, Sichuan 610041, PR China, ${ }^{2}$ Department of Respiratory Medicine, The $7^{\text {th }}$ People's Hospital, Chengdu, Sichuan 610041, PR China, ${ }^{3}$ Department of Functional Branch, The Leshan People's Hospital, Leshan, Sichuan 614000, PR China and ${ }^{4}$ Laboratory of Pulmonary Disease, West China Hospital, Sichuan University, Chengdu, Sichuan 610041, PR China.

Correspondence: Dr C Liu, Department of Respiratory Medicine, West China Hospital, Sichuan University, Chengdu, Sichuan 610041, PR China. Fax: +8628 85423872, e-mail: LuoBao97@163.com
(1). Although $\beta$-agonists acutely improve lung function, chronic use impairs therapeutic efficiency and even induces serious adverse effects (2). Compelling evidence confirms the findings of earlier studies that there is an increase in asthma-related mortality with chronic use of $\beta$-agonists (3).

More and more clinical studies report that regular use of $\beta$-agonists induces loss of bronchoprotection (4-6). The mechanisms of $\beta_{2}$-adrenoceptors that underlie loss of bronchoprotection have not been elucidated thus far. Bronchial smooth muscle (BSM) is not only the main effector of bronchial contraction, but has also been considered as an inflammatory cell per se (7). As an inflammatory cell, BSM can worsen bronchial inflammation by secreting inflammatory mediators, recruiting and activating inflammatory cells including T-lymphocytes and mast cells. Bronchial smooth muscle mass is dramatically increased in asthma, which is characterized by an increased BSM cell number (hyper- 
plasia), an increased BSM cell size (hypertrophy), and an increased deposition of extracellular matrix proteins collagen - in and around the BSM bundles (6). Increased BSM mass contributes significantly to overall airway remodelling and response to various stimuli; it also correlates with decline in lung function and severity of asthma. Smooth muscle (SM) $\alpha$-actin is the first smooth muscle cell differentiation marker to appear during development and is also expressed in fibroblast differentiation to smooth muscle cell during airway remodelling or repairing; moreover, its expression is primarily restricted to smooth muscle cell in adult animals. Therefore, $\alpha$-actin is the ideal marker of smooth muscle cells (8).

Thus far, whether impaired bronchoprotection induced by formoterol is caused by increased BSM mass has not been reported. The goal of this study is to establish a mice model that reproduced the loss of bronchoprotection induced by formoterol observed in some asthmatics. We will then test our hypothesis that formoterol-induced impaired bronchoprotection is caused by increased BSM mass, through airway morphometric analysis and expression of SM $\alpha$-actin (immunohistochemistry and western blot).

\section{SUBJECTS AND METHODS}

All mice care and experimental protocols were examined and approved by the Institutional Animal Care and Use Committee at Sichuan University. A total of 60 specific pathogenfree, eight-week old female BALB/c mice, weight 20-25 g, were purchased from the Experiment Animal Centre in Sichuan Academy of Medical Sciences. All the mice were kept in a pathogen-free barrier facility.

\section{Drug formulations}

The formoterol fumarate dihydrate (F ORM) was bought from Sigma in the United States of America (USA). The vehicle of the drugs was composed by (w/v): EDTA $(0.1 \%)$, sodium chloride $(8.5 \%)$, citric acid dried $(0.15 \%)$, polysorbate $80(0.2 \%)$, sodium citrate $(0.5 \%)$ in deionized water. Formoterol fumarate dihydrate formulation was homogenized in polysorbate 80 and deionized water. Budesonide (BUD)/F ORM compound was made by adding BUD to F ORM suspension to the concentrations of $200 \mathrm{mg} / \mathrm{ml}$ and $5.72 \mathrm{mg} / \mathrm{ml}$, respectively. All formulations were at $\mathrm{pH} 5.0$ and kept away from light. The intratracheal instillation volumes were $1 \mathrm{ml} / \mathrm{kg}$ body weight in all mice. The BUD/ F ORM ratio was adjusted to the ratio $35: 1$ based on the clinical formulation (9) and experiment performed previously in rats (10).

\section{Mouse with chronic ovalbumin (OVA) and FORM treatment}

Mice were sensitized on days zero and 14 through intraperitoneal injection of $20 \mathrm{mg}$ ovalbumin (OVA; Sigma, USA) with $2 \mathrm{mg}$ of aluminum adjuvant diluted in saline, as described before (11). Beginning on day 18 and continuing until day 49, mice were challenged for 60 minutes, five days in the first week and subsequently three days per week, with four rest days in between, by a 1\% OVA aerosol produced by an atomizer (PARI, Germany). Two hours after OVA challenging, mice were modestly anaesthetized with isoflurane and then $20 \mu \mathrm{l} \mathrm{FORM}(0.29 \mu \mathrm{g} / \mathrm{kg})$, BUD $(10 \mu \mathrm{g} / \mathrm{kg})$ plus FORM or vehicle $(0.9 \%$ saline $)$ was dropped into the trachea, respectively, after a maximal oropharyngeal exposure by a specific mouse laryngoscope. Animals were tested 24 hours after the last challenging day of each week. The FORM was delivered by oropharyngeal instillation to imitate the method of delivery in humans and thereby avoid potentially adverse effects of systemic FORM use, as shown in Fig. 1 (12).

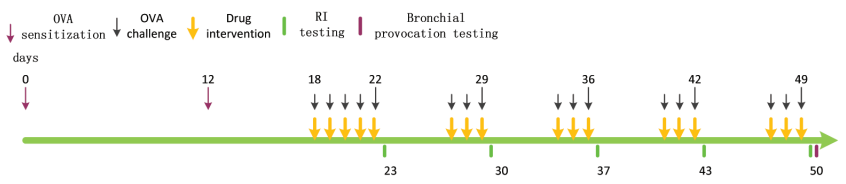

Fig. 1: Schematic overview of study protocol for induction of prolonged allergen challenge and therapeutic administration of drugs. OVA: ovalbulmin; RI: airway resistance

\section{Evaluation of airway responsiveness: impedance}

Airway responsiveness was measured 24 hours after the last challenging day of each week and mice were anaesthetized and paralysed with intraperitoneal $80 \mathrm{mg} / \mathrm{kg}$ sodium pentobarbital and $0.25 \mathrm{mg} / \mathrm{kg}$ pancuronium bromide, respectively. Once mice were anaesthetized, a tracheal cannula and special-made caudal vein catheter were inserted and fixed with sutures. The animal was ventilated (Buxco, USA) at a constant volume of $7 \mathrm{~mL} / \mathrm{kg}$ and a frequency of 160 breaths/ minute. We firstly established that the dose of methacholine (MCh) administered was maximal (400 $\mu \mathrm{g} / \mathrm{kg})$, which was not lethal for the mice, and defined the airway response to MCh by the maximal airway resistance (RI) value (RImax). The mice were ventilated until airway resistance was maintained stable for two minutes before each injection of drug which was injected after five-minute washing intervals. In brief, MCh, $400 \mu \mathrm{g} / \mathrm{kg}$ and $35 \mu \mathrm{l}$ for each mouse, was injected first, followed by terbutaline $(30 \mu \mathrm{g} / \mathrm{kg}$ and $35 \mu \mathrm{l}$ for each mouse) and MCh. Injection of terbutaline/MCh was repeated three times. Airway resistance measurements (RImax and basal RI) were collected before and after each drug injection (Fig. 2).

\section{FORM-mediated bronchoprotection protocol}

The RImax response to $\mathrm{MCh}$ and combined terbutaline/MCh was recorded. Bronchoprotection was calculated as the percentage change in impedance by the equation: [/RImax response to $M C h$ - (RImax response to terbutaline/MCh)]/ RImax response to $M C h]$. We analysed the bronchoprotective effect mediated by terbutaline in five consecutive weeks. 


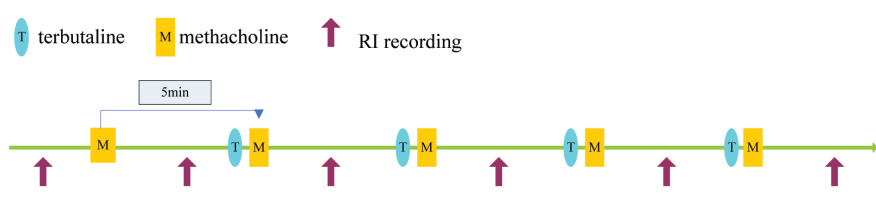

Fig. 2: Schematic overview of study protocol for administration of drugs and detecting airway resistance (RI) .

\section{Haematoxylin and eosin (H\&E) staining}

Lung sections were stained by H\&E for two reasons: first, to see whether an asthmatic model was successfully established, assessed by two points - perivascular or peribronchiolar inflammation in low magnification; and second, to compare the depth of BSM in each group in high magnification.

\section{Airway structural changes by morphometric analysis (collagen, SM $\alpha$-actin)}

Masson-trichrome stain was used to stain lung sections for elastin collagen. The area among the outer extent of the total collagen layer in the submucosal region and the basement membrane was measured to semi-quantitate the thickness of airway wall collagen. The length of basement membrane was measured to normalize the detected area (13). To identify SM $\alpha$-actin, mouse monoclonal to $\alpha$-actin was used with a biotinylated goat-anti-mouse secondary antibody. ImagePro Plus Software was applied to semi-quantitatively evaluate SM $\alpha$-actin. Smooth muscle $\alpha$-actin positive cells in only bronchi were counted by the ratio of integrated option density (IOD) and the detecting area.

\section{Smooth muscle $\alpha$-actin expression (western blot)}

For western blot analysis, samples were lysed in RIPA (radioimmunoprecipitation assay), adding PMSF (phenylmethylsulfonyl fluoride) and separated on a 10\% SDS-PAGE (sodium dodecyl sulfate polyacrylamide gel electrophoresis) gel, followed by western blotting with 1:1000 rabbit antimouse $\alpha$-actin (Santa Cruz Biotechnology, USA) and $\beta$-actin (Abcam, USA). Secondary antibody - horseradish peroxidase conjugated horse anti-rabbit antibody (CST, USA) with dilution 1:6000 - was applied for chemiluminescence.

\section{Statistical analysis}

Data are expressed as mean \pm SEM. Statistical analysis was performed using SPSS 10. Significant differences among groups were identified by analysis of variance (ANOVA). A $p$-value of less than 0.05 was considered statistically different.

\section{RESULTS}

\section{Airway responsiveness}

As shown in Fig. 3A, chronic FORM treatment resulted in a significant increase in RImax response to $\mathrm{MCh}$, relative to the two other groups in the last week $(p<0.05)$, while no significant difference was detected in the controls and

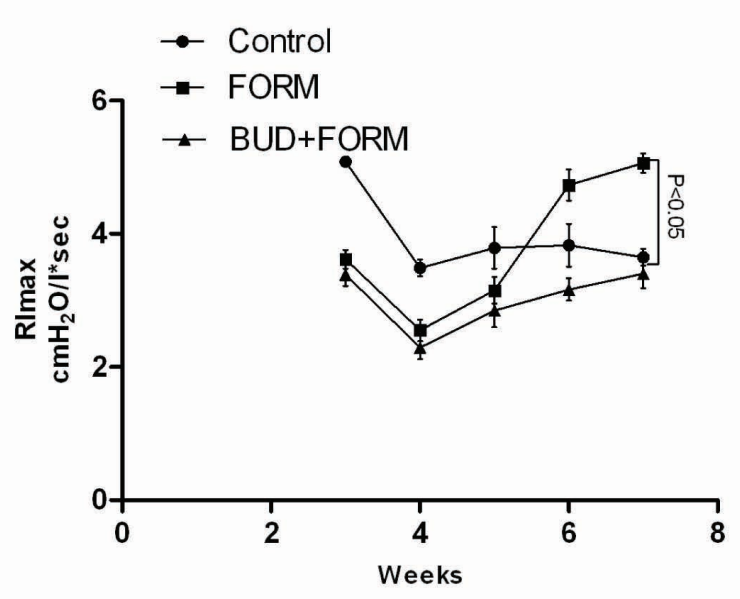

Fig. 3A: Maximal airway resistance (RImax) induced by methacholine measured in five consecutive weeks.

FORM: formoterol fumarate dehydrate; BUD: budesonide

BUD+FORM group $(p>0.05)$. Animals treated with FORM as well as those in the BUD+FORM group showed a wave trend of RImax - a decline in the first two weeks and then a continuous increase. The control group had the highest RImax in the third week; after that, RImax declined and stayed at a relatively stable level. Although adding BUD significantly inhibits RImax, there was also a modest increased trend in the last three weeks. Accordingly, mice chronically treated with FORM demonstrated increased airway responsiveness.

\section{Bronchoprotection}

The bronchoprotective effect of $\beta$-agonists was significantly impaired in mice chronically treated with FORM (Fig. 3B).

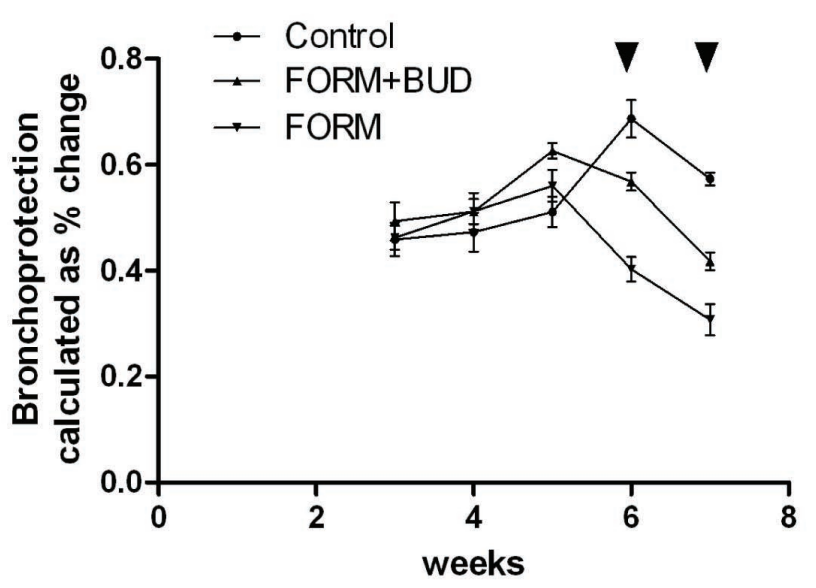

Fig. 3B: Bronchoprotection calculated as a percentage change in methacholine-induced impedance in five consecutive weeks. Black triangle means significant differences were detected $(p<0.05)$ in each two groups. 
Consistent with airway responsiveness, the per cent change in bronchoprotection was wavy - it increased in the first three weeks and then declined in the last two weeks in the FORMtreated group as well as the BUD+FORM group. The FORM-group had the lowest effect of bronchoprotection, while the control group had the highest level in the last two weeks $(p<0.05)$. This meant that in the fifth week, FORM exerted maximal bronchoprotection, after which the effect of bronchoprotection was impaired. Our mouse model successfully reproduced the association between chronic long-acting $\beta$-agonist use and $\beta$-adrenoceptor dysfunction, or tolerance observed in some asthmatics.

\section{Haematoxylin and eosin staining}

This mouse model of chronic allergen challenging manifested many other characteristics of human asthmatics. The control group demonstrated enhanced peribronchiolar and perivascular inflammation accompanied by epithelial shedding and epithelial layer damage. The administration of FORM to the chronic OVA challenging mice caused a significant increase in peribronchiolar and perivascular inflammation. Chronic treatment with FORM plus BUD significantly inhibited the inflammatory reactivity. There was no significant difference in increased BSM mass among each group according to the high magnification figure $(\times 600)$, as seen in Fig 4.

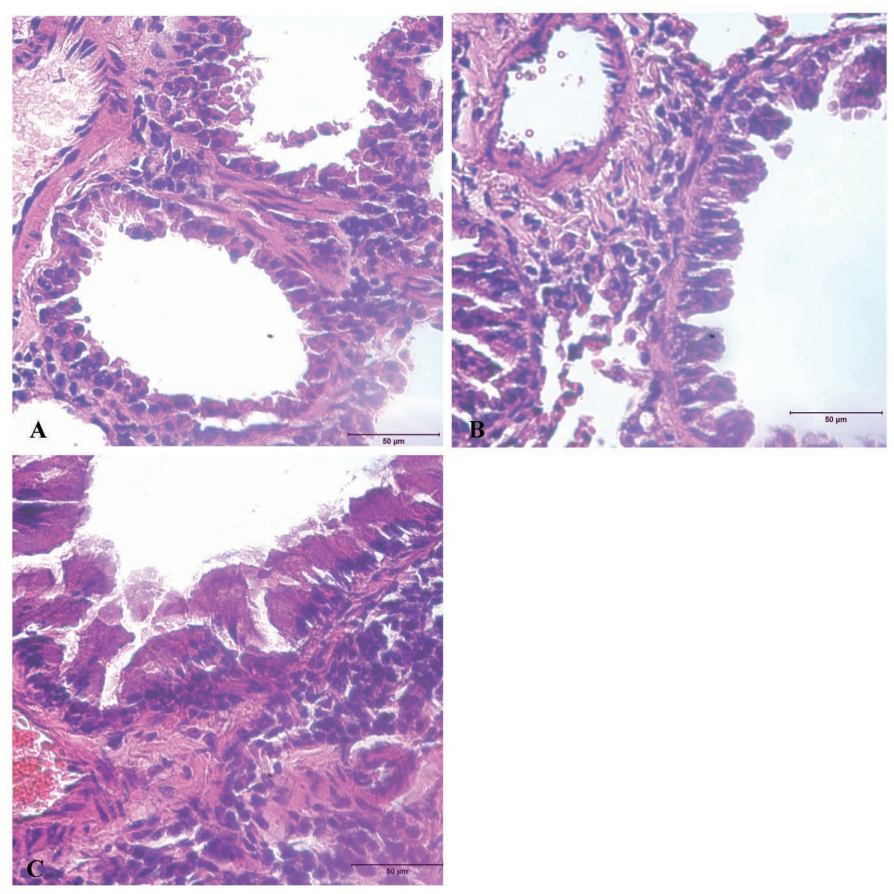

Fig. 4: Lung slides stained with haematoxylin and eosin (magnification $\times 600$ ). A: Control group. B: Formoterol fumarate dehydrate (FORM) group. C: FORM plus budesonide (BUD) group. There was no significant difference in increased bronchial smooth muscle mass in each group.

\section{Collagen deposition and SM $\alpha$-actin expression}

Chronic FORM treatment caused no increase in subepithelial collagen deposition and no statistical difference was detected in each of the groups $(p>0.05)$ [Fig. 5A-D]. To assess mor-
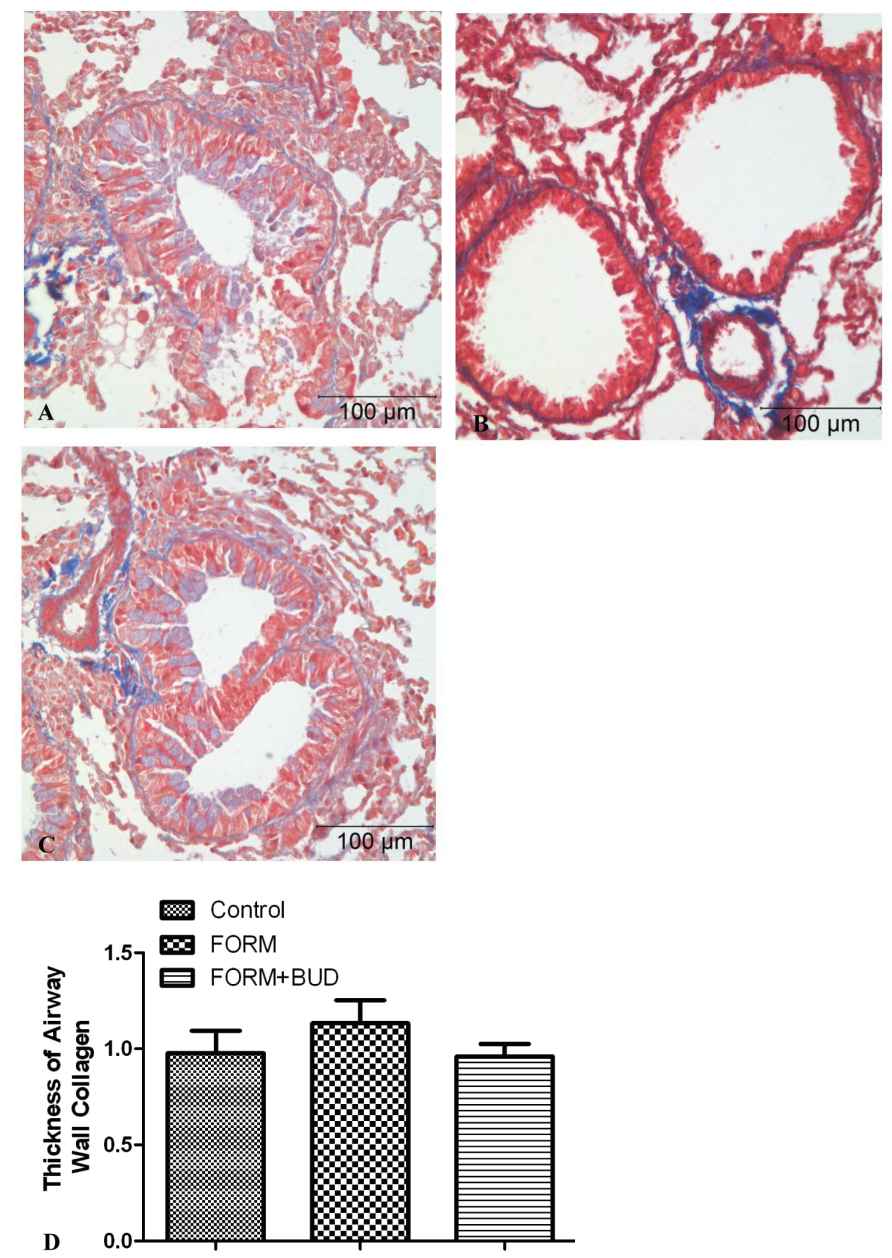

Fig. 5: Assessment of depth of airway collagen (Masson-trichrome staining, magnification $\times 400$ ). A: Control group. B: Formoterol fumarate dehydrate (FORM) group. C: FORM plus budesonide (BUD) group. D: There was no significant difference in collagen deposition in each group $(p>0.05)$.

phological remodellings in bronchial smooth muscle, slides were semi-quantitated by the ratio of smooth muscle $\alpha$-actin staining. We found no effect of chronic FORM or BUD administration on the volume of smooth muscle around bronchi $(p>0.05)$ [Fig. 6A-D].

Smooth muscle $\alpha$-actin expression level was analysed by western blot (Fig. 7). Alpha-actin in the FORM group (Lane 2) showed no difference as compared with that in the control (Lane 1) and BUD (Lane 3) groups. That meant that neither FORM nor BUD could promote BSM mass; thus we concluded that impaired bronchoprotection induced by chronic use of FORM was not caused by increased BSM mass. 

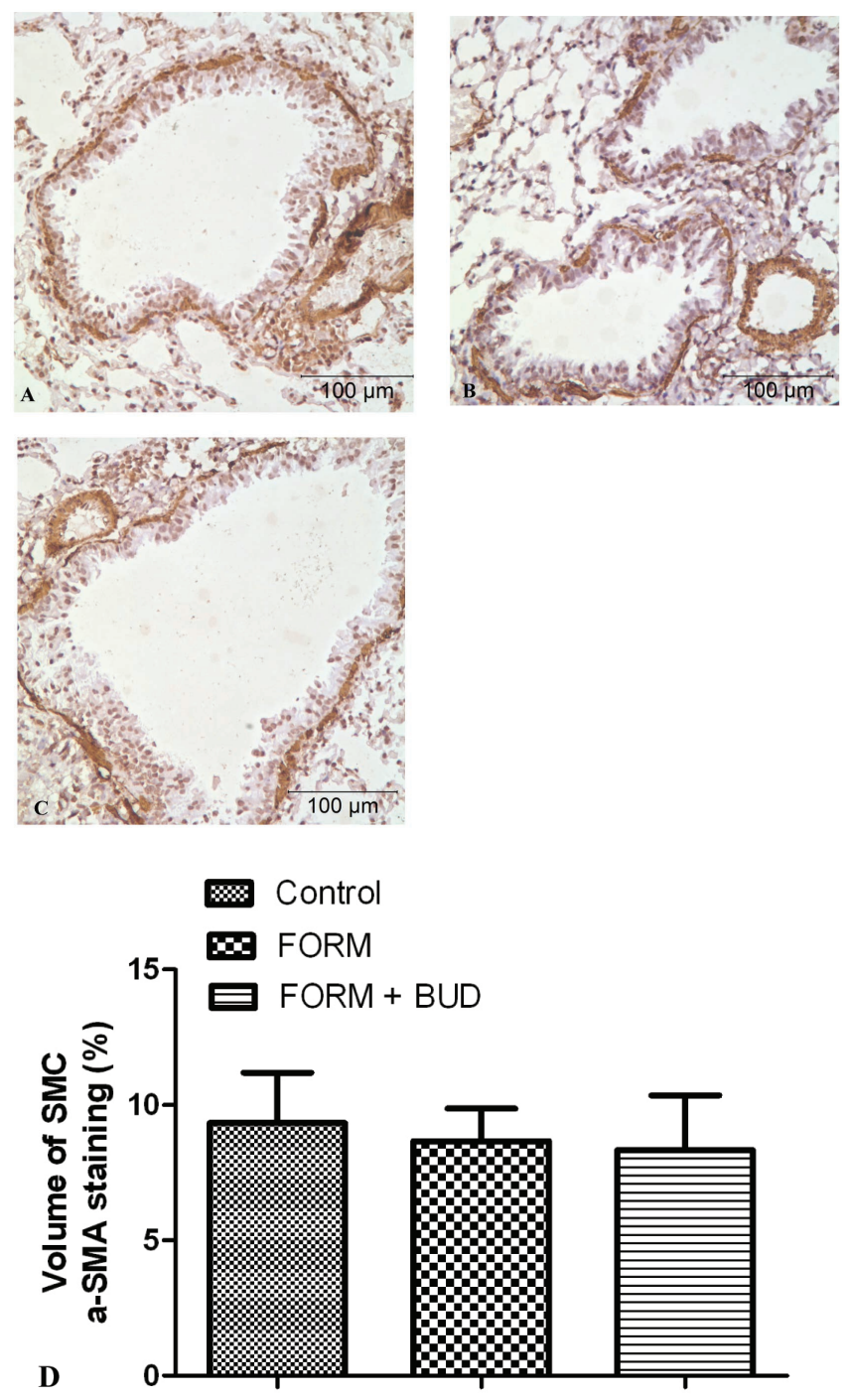

Fig. 6: Amount of airway smooth muscle cells including hypertrophy and hyperplasia. A: Control group ( $\alpha$-SMA immunohistochemisty, magnification $\times 400$ ). B: Formoterol fumarate dehydrate (FORM) group. C: FORM plus budesonide (BUD) group. D: There was no significant difference in each two groups.

1

2

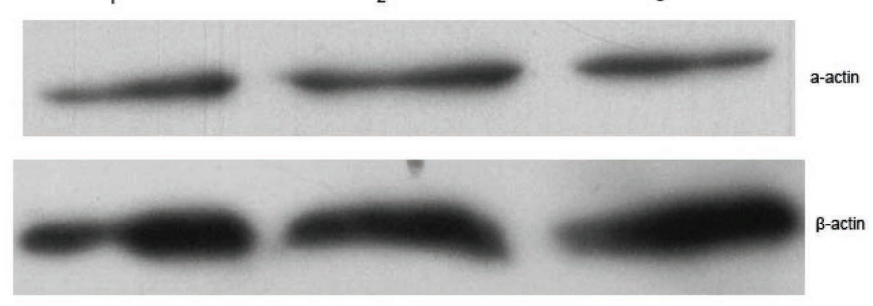

Fig. 7: Detection of $\alpha$-smooth muscle actin ( $\alpha$-SMA) expression in the lung. Lung tissues were collected and analysed by western blot. Lane 1: Control group; Lane 2: Formoterol fumarate dehydrate (FORM) group; Lane 3: FORM plus budesonide (BUD) group.

\section{DISCUSSION}

Beta-agonists are first-line therapeutic drugs for the treatment of asthma, especially the drug containing FORM and BUD such as Symbicort Turbuhaler ${ }^{\circledR}$. However, when used regularly, their effectiveness declines, and they might gradually enhance disease progression. This article firstly provides a detailed description of the effects of chronic formoterol administration in the chronic allergen challenging airway inflammatory disease and dynamic changing of bronchoprotection in seven consecutive weeks.

This finding is crucial because it proves that chronic administration of formoterol can exacerbate airway inflammation and responsiveness and impair bronchoprotection of $\beta$-agonists. Accordingly, $\beta_{2}$-adrenoceptor dysfunction might be an underlying mechanism of worsening airway inflammation. Thus far, only two animal studies have evaluated the effect of chronic $\beta$-agonist treatment on bronchoprotection; however, only indirect reflection of bronchoprotection and short-acting $\beta$-agonists were included in these trials $(14,15)$. Efforts to research the underlying mechanisms of $\beta_{2^{-}}$adrenoceptor dysfunction and relevant adverse events have been hindered by human ethic limitations and lack of animal models that combine the optimal route, dose and duration of $\beta$-agonist administration with sufficient duration of allergen challenging. The chronic FORM treatment that we applied in mice successfully demonstrated increased airway responsiveness and impaired bronchoprotection observed in asthmatics.

More and more trials have strongly demonstrated that excess smooth muscle, including hyperplasia and hypertrophy, is present in the airways of patients with asthma. Tissue remodelling due to increased BSM mass in allergic asthma is also known to correlate with airway hyper-responsiveness and severity of asthma in patients. Taken together, smooth muscle amount, and changes in its mass are likely to be key players in the dynamics of airway narrowing, including increased airway hyper-responsiveness and basic bronchial tension $(6,8)$. However, according to the morphometric analysis of structure changes - collagen deposition, $\alpha$-smooth muscle actin expression - impaired bronchoprotection induced by chronic FORM treatment seems to have no relevance with increased smooth muscle mass. That means that chronic treatment with FORM or FORM+BUD will not worsen or attenuate BSM hyperplasia or hypertrophy. Although no significant BSM mass changes were observed in the chronic FORM-treatment group, BSM is still the most important research target. Future research might focus on whether receptor modification or altered signalling, such as $\mathrm{Ca}^{2+}$ mobilization or $\mathrm{Ca}^{2+}$ sensitization, of BSM is involved. In fact, more work has been done to reveal the molecular mechanisms of impaired bronchoprotection in our laboratory based on the model, and more data will be published in upcoming articles. 


\section{CONCLUSION}

The animal model provides a useful tool to facilitate relevant research, especially the chronic effects of other $\beta$-adrenoceptor agonists and the underlying mechanism of $\beta$-adrenoceptor dysfunction. Secondly, we demonstrated that chronic FORM treatment can promote peribronchiolar and perivascular lung inflammation and impair bronchoprotection. Thirdly, impaired bronchoprotection induced by FORM is not caused by increased BSM mass.

\section{ACKNOWLEDGEMENT}

The authors gratefully received support from the Natural Science Foundation of China (grant 81170031) and the Laboratory of Pulmonary Disease, West China Hospital, Sichuan University.

\section{REFERENCES}

1. Abisheganaden J, Boushey HA. Long-acting inhaled beta 2-agonists and the loss of "bronchoprotective" efficacy. Am J Med 1998; 104: 494-7.

2. Taylor DR. The beta-agonist saga and its clinical relevance: on and on it goes. Am J Respir Crit Care Med 2009; 179: 976-8.

3. Aaronson DW. The "black box" warning and allergy drugs. J Allergy Clin Immunol 2006; 117: 40-4

4. Drotar DE, Davis EE, Cockcroft DW. Tolerance to the bronchoprotective effect of salmeterol 12 hours after starting twice daily treatment. Ann Allergy Asthma Immunol 1998; 80: 31-4.

5. Jokic R, Swystun VA, Davis BE, Cockcroft DW. Regular inhaled salbutamol: effect on airway responsiveness to methacholine and adenosine 5 '-monophosphate and tolerance to bronchoprotection. Chest 2001; 119: 370-5.

6. Bara I, Ozier A, De Lara JT, Marthan R, Berger P. Pathophysiology of bronchial smooth muscle remodelling in asthma. Eur Respir J 2010; 36: 1174-84.

7. Berger P, Girodet PO, Manuel Tunon-de-Lara J. Mast cell myositis: a new feature of allergic asthma? Allergy 2005; 60: 1238-40.
8. Al-Muhsen S, Johnson JR, Hamid Q. Remodeling in asthma. J Allergy Clin Immunol 2011; 128: 451-62.

9. Pauwels RA, Löfdahl C-G, Postma DS, Tattersfield AE, O’Byrne P, Barnes PJ et al. Effect of inhaled formoterol and budesonide on exacerbations of asthma. N Engl J Med 1997; 337: 1405-11.

10. Brange C, Smailagic A, Jansson A-H, Middleton B, Miller-Larsson A, Taylor JD et al. Sensitivity of disease parameters to flexible budesonide/formoterol treatment in an allergic rat model. Pulm Pharmacol Ther 2009; 22: 20-6.

11. Walker JK, Fong AM, Lawson BL, Savov JD, Patel DD, Schwartz DA et al. Beta-arrestin-2 regulates the development of allergic asthma. J Clin Invest 2003; 112: 566-74.

12. McMillan S, Xanthou G, Lloyd C. Therapeutic administration of budesonide ameliorates allergen-induced airway remodelling. Clin Exp Allergy 2005; 35: 388-96.

13. Veness-Meehan KA, Pierce RA, Moats-Staats BM, Stiles AD. Retinoic acid attenuates O2-induced inhibition of lung septation. Am J Physiol Lung Cell Mol Physiol 2002; 283: L971-80.

14. Callaerts-Vegh Z, Evans KL, Dudekula N, Cuba D, Knoll BJ, Callaerts $\mathrm{PF}$ et al. Effects of acute and chronic administration of betaadrenoceptor ligands on airway function in a murine model of asthma. Proc Natl Acad Sci 2004; 101: 4948-53.

15. Lin R, Degan S, Theriot BS, Fischer BM, Strachan RT, Liang J et al. Chronic treatment in vivo with beta-adrenoceptor agonists induces dysfunction of airway beta (2) -adrenoceptors and exacerbates lung inflammation in mice. Br J Pharmacol 2012; 165: 2365-77.

Submitted 24 Jul 2014

Accepted 11 Aug 2014

Published 22 Sep 2014

Online: http://myspot.mona.uwi.edu/wimjopen/article/1597

(C) Luo et al 2014.

This is an open access article made freely available under Creative Commons Attribution 4.0 International (CC BY 4.0). Users are free to share, copy and adapt this work as long as the copyright holder (author) is appropriately and correctly credited. See http://creativecommons. org/licences/by/4.0/deed.en_us for more information. 